\title{
Linx
}

Revue des linguistes de l'université Paris X Nanterre

$72 \mid 2015$

Former à l'écrit universitaire, un terrain pour la linguistique?

\section{Dispositifs didactiques en littératie universitaire : le cas du Centre d'aide en français écrit à l'Université du Québec en Outaouais}

\author{
Lizanne Lafontaine, Judith Emery-Bruneau et Amélie Guay
}

\section{OpenEdition}

\section{Journals}

Édition électronique

URL : http://journals.openedition.org/linx/1590

DOI : $10.4000 /$ linx.1590

ISSN : 2118-9692

\section{Éditeur}

Presses universitaires de Paris Nanterre

Édition imprimée

Date de publication : 1 septembre 2015

Pagination : $39-54$

ISSN : 0246-8743

\section{Référence électronique}

Lizanne Lafontaine, Judith Emery-Bruneau et Amélie Guay, « Dispositifs didactiques en littératie universitaire : le cas du Centre d'aide en français écrit à l'Université du Québec en Outaouais », Linx [En ligne], 72 | 2015, mis en ligne le, consulté le 19 avril 2019. URL : http://journals.openedition.org/ $\operatorname{linx} / 1590 ; \mathrm{DOl}:$ : 10.4000/linx.1590 


\title{
Dispositifs didactiques en littératie universitaire : le cas du Centre d'aide en français écrit à l'Université du Québec en Outaouais
}

\author{
Lizanne LAFONTAINE, Judith EMERY-BRUNEAU et Amélie GUAY \\ Université du Québec en Outaouais (UQO), Canada
}

Résumé

Cet article présente la problématique de la littératie universitaire et recense les approches en pédagogie universitaire mises en place dans un CAFÉ (Centre d'Aide en Français Écrit), en s'appuyant sur le cas du CAFÉ de l'Université du Québec en Outaouais (UQO). Après avoir dressé le portrait des mesures de remédiation en littératie universitaire mises en place à l'UQO et observé leurs effets sur l'augmentation du taux de réussite à une épreuve de certification nationale en français écrit, nous concluons qu'une analyse plus approfondie doit être réalisée. Les étudiants fréquentant le CAFÉ semblent avoir amélioré leur compétence langagière, mais au-delà du fait de réussir un test, nous ne connaissons pas l'évolution de leur rapport à l'écrit dans un contexte particulier de remédiation. Cet aspect de la recherche en littératie universitaire est non négligeable et fera l'objet de travaux inscrits en continuité de cette modeste contribution qui ouvre la voie à un chantier encore peu exploré.

Mots-clés

Littératie, littératie universitaire, dispositifs didactiques, remédiation en français écrit, centre d'aide en français écrit.

Les enquêtes internationales soutenues par l'OCDE $(2004,2011)$ et celle du Réseau canadien de recherche sur le langage et l'alphabétisation (2009) indiquent que la majorité des adolescents et des jeunes adultes québécois ne maitrisent ${ }^{1}$ pas, en lecture, les habiletés supérieures nécessaires pour atteindre un haut niveau de littératie complexe. Or, la compétence à écrire en français est un élément essentiel à la réussite sociale et scolaire. De surcroit, la maitrise du français écrit représente souvent un fardeau pour les étudiants postsecondaires québécois qui n'arrivent pas à atteindre un niveau suffisant au Test de certification en français écrit pour l'enseignement (TECFEE), certification nationale dont la réussite est obligatoire pour obtenir leur diplôme et leur brevet d'enseignement. Un échec à ce test peut considérablement ralentir leurs études universitaires et les universités misent sur les centres d'aide en français écrit (CAFÉ) afin de remédier en partie au problème (Nadeau, 2010). Nous présenterons quelques résultats d'une préenquête décrivant les retombées importantes de certains dispositifs didactiques sur la maitrise du français écrit d'étudiants universitaires. Ces dispositifs traitent des éléments linguistiques suivants : grammaire de la phrase, grammaire du texte, orthographe lexicale, ponctuation, genres et modes du discours universitaires.

\section{Problématique : la littératie en contexte universitaire}

Hébert et Lépine (2012) ont réalisé une étude faisant état d'une centaine d'articles écrits depuis 2000 définissant la littératie en contexte francophone. À la lumière de leur recension, ils

\footnotetext{
${ }^{1}$ Ce texte adopte l'orthographe rectifiée.
} 
identifient 10 valeurs ajoutées de la littératie ${ }^{2}$, mais concluent que cette notion est difficile à définir. Force est de constater que la notion de littératie universitaire l'est encore plus. En effet, peu de recherches la définissent. L'expression est surtout employée comme terme générique faisant référence à l'écrit et aux discours universitaires (Barbier, 2009, Pollet, Glorieux et Toungouz, 2010, Boeglin et Jean, 2011, Lampron et Blaser, 2012); on y fait état de la difficulté des étudiants universitaires à rédiger des écrits tels que des dissertations, des mémoires, des thèses. Cependant, l'absence de maitrise des discours universitaires ne devrait pas être interprétée comme un déficit des étudiants ; c'est à l'université de les enseigner (Delcambre et LahanierReuter, 2010). En outre, la plupart des diplômés universitaires se dirigent vers des professions où ils devront être des rédacteurs capables de produire des documents professionnels complexes qui respectent les conventions de leur milieu (Labasse, 2006). Ces états de fait confirment le constat voulant que la société du savoir soit essentiellement une société de l'écrit et de l'écriture (HartDavidson et Grabill, 2011). Pourtant, la plupart des professionnels formés dans les universités, dont ceux qui se destinent à l'enseignement, ne reçoivent que peu ou pas de formation en communication écrite (Beaudet et Rey, 2012).

\subsection{Les difficultés des étudiants universitaires en écriture}

Au Québec, depuis 2008, tous les étudiants en formation initiale à l'enseignement (FIE) doivent réussir un test de français national, le TECFÉE ${ }^{3}$, formé de deux parties ${ }^{4}$. Chaque partie est réalisée en temps limité ${ }^{5}$ et doit être réussie avec la note minimale de $70 \%{ }^{6}$. En 2007, à la validation finale, le taux de réussite était de moins de 50\%. En 2008, le taux de réussite provincial variait de $20 \%$ à $59 \%$ selon les universités. Or, les conséquences à la suite d'un troisième échec sont la suspension de la formation en enseignement afin de suivre une formation non créditée de mise à niveau en grammaire et en français écrit ${ }^{7}$. Le besoin de remédiation en français écrit de nos étudiants est donc bien réel.

\subsection{Problématique : les mesures de remédiation existantes}

\footnotetext{
${ }^{2}$ Voici la liste des 10 valeurs ajoutées à la notion : pluri-objectifs; ensemble d'attitudes, de connaissances, de compétences et d'habiletés liées à l'appropriation de la culture écrite; variété des textes, des genres, des supports; aspect dynamique, variable et situé; relations individu-société; tâches réelles authentiques, scolaires et extrascolaires; concept positif et continuum; interdépendance-interférence écrit-oral; influence de l'environnement; visée émancipatrice.

3 Avant 2003, les étudiants en FIE devaient réussir un test de français spécifique à chaque université. De 2003 à 2008, ils devaient réussir un autre test de français administré par un des organismes nationaux suivants : Service d'évaluation linguistique (SEL) ou Centre d'évaluation du rendement en français écrit (CEFRANC).

${ }^{4}$ Ce test est formé de deux parties : des questions à choix multiple portant sur le code linguistique et la rédaction d'un compte-rendu critique de 300 mots produit à partir de l'écoute d'un document sonore.

5 Pour le questionnaire à choix multiple, aucun ouvrage de référence n'est autorisé. Pour la rédaction, un seul ouvrage de référence, par exemple une grammaire ou un dictionnaire, est autorisé. Le temps de rédaction est organisé comme suit : $1 \mathrm{~h} 30$ pour les questions à choix multiple et $2 \mathrm{~h} 30$ pour la rédaction).

${ }^{6}$ En enseignement de l'anglais langue seconde et en enseignement professionnel et technique, le seuil de réussite est fixé à $55 \%$.

${ }^{7}$ Selon les universités, les conséquences peuvent changer. Par exemple, dans notre université, ceux qui y échouent après une troisième tentative doivent suivre une formation grammaticale non créditée de quatre cours et les réussir avec la note minimale de $\mathrm{B}+$ afin d'être admis de nouveau dans les programmes de FIE.
} 
Toutes les universités québécoises proposent de la remédiation en lien avec la langue écrite. Or, c'est l'ordre collégial ${ }^{8}$ qui a surtout contribué au développement des mesures de remédiation. En effet, Moffet et Demalsy (1994) nous apprennent que toutes les institutions collégiales québécoises $(n=37)$ offrent de telles mesures. Ces mesures existent principalement sous deux formes : des cours de mise à niveau (33/37) et des CAFÉ (36/37). Les cours de mise à niveau portent surtout sur la grammaire de la phrase et, parfois, sur la lecture et la rédaction. Quant aux CAFÉ, ils sont apparus au milieu des années 1980, alors que les collèges québécois étaient préoccupés par la qualité de la langue des étudiants (Claing, 2007). Le tutorat individuel est la méthode privilégiée : il est assuré par des pairs, des étudiants universitaires ou, plus rarement, par des enseignants.

À l'instar du collégial, les universités offrent, depuis les années 2000, de la remédiation dans des CAFÉ afin d'intervenir de façon plus individuelle et ponctuelle (Beaudry, Camerlain et Bégin, 2007). Les mesures de remédiation proposées varient grandement selon les institutions. Parmi les plus fréquentes et les plus populaires, nous retrouvons les rencontres individuelles et les ateliers thématiques autour de notions grammaticales (Beaudry, Camerlain et Bégin, 2007, Lefrançois, Laurier, Lazure et Claing, 2008; Cartier et Langevin, 2001). Certains CAFÉ offrent aussi des services de consultation libre, des exercices en ligne et des ateliers consacrés à la préparation de tests de français, dont le TECFÉE. Par les objectifs qu'ils poursuivent, les CAFÉ s'inscrivent dans la tradition des «writing centers » américains, que l'on retrouve aussi ailleurs dans le monde ${ }^{9}$.

Alors que les études au sujet des «writing centers », qui existent depuis plus de 30 ans, sont nombreuses, l'efficacité de la remédiation proposée par les CAFÉ en contexte francophone a été peu abordée. Parmi les études réalisées, celle de Cartier et Langevin (2001) révèle que les mesures d'aide les plus populaires sont les ateliers thématiques autour de notions grammaticales ciblées et l'aide individuelle. Par ailleurs, Lefrançois, Laurier, Lazure et Claing (2008) concluent que l'aide individuelle (dans un CAFÉ) a permis à des étudiants en FIE de diminuer de 42,83\% leurs erreurs d'orthographe lexicale. Ces chercheurs concluent également que certaines mesures sont plus efficaces pour remédier à des lacunes spécifiques. Ainsi, une offre diversifiée qui combine différentes mesures s'avèrerait bénéfique afin de répondre aux besoins des étudiants et permettrait à ces derniers d'améliorer leur français écrit de façon plus «rentable » en termes de temps et d'efforts.

Par ailleurs, il ne faut pas oublier que la situation des étudiants en FIE qui éprouvent des difficultés en écriture est doublement complexe. En effet, maitriser la langue ne suffit pas ; ils doivent aussi être en mesure de l'enseigner, de la promouvoir et de corriger les productions des élèves. Confrontées à cette réalité, il nous semble opportun d'adopter l'approche globale que les concepts de littératie et de littératie universitaires proposent.

\section{Cadre théorique : les concepts de littératie et de littératie universitaire}

\subsection{La littératie}

\footnotetext{
${ }^{8}$ L'ordre collégial se trouve entre le secondaire et l'ordre universitaire (étudiants de 17 à 19 ans).

${ }^{9}$ L'International Writing Centers Association (IWCA) regroupe des centres se trouvant dans plus d'une vingtaine de pays.
} 
La littératie est une compétence générale qui permet à toute personne d'avoir accès au monde extérieur, d'interagir, de communiquer, d'apprendre, de se socialiser et d'effectuer des calculs simples. Elle comprend les nombreuses formes de littératies requises pour réussir dans nos sociétés basées sur le savoir comme la lecture, l'écriture, l'expression orale, le visionnement de documents sonores et la représentation (Grenier, Jones, Strucker, Murray, Gervais et Brink, 2008) ainsi que l'analyse d'informations, la compréhension de concepts abstraits et l'acquisition de nombreuses autres compétences complexes (Conseil canadien sur l'apprentissage, 2011).

De manière plus précise, la littératie met en œuvre des habiletés complexes que sont la lecture et l'écriture. De plus, selon Moreau, Hébert, Lépine et Ruel (2013), la littératie reflète les valeurs et permet le développement d'attitudes, de comportements et de savoirs liés à la culture de l'écrit. Ainsi, ce concept illustre la manière dont une personne comprend et transmet des informations simples ou complexes dans des situations de communications orale et écrite, et dans une variété de supports imprimés ou non (Coghlan et Thériault, 2002). Elle est source d'apprentissage, d'information, de communication, de développement identitaire et culturel. C'est un concept aux objectifs multiples, souvent interdisciplinaires, permettant de toucher à la fois les sphères personnelle, professionnelle et socioculturelle ou écologique liées à l'apprentissage de l'écrit (Moreau et al, 2013).

\subsection{La littératie universitaire}

Très peu de recherches ont défini le concept de littératie universitaire. Toutefois, un constat émerge : la littératie universitaire représente les genres et les modes de discours universitaires ainsi que les difficultés rencontrées chez les étudiants dans leur mise en pratique. Ces productions sont différentes selon les disciplines universitaires et ont chacune leurs propres caractéristiques qui doivent être enseignées, ce qui oblige par la même occasion à proposer des mesures de remédiation aux étudiants (Barbier, 2009, Delcambre et Lahanier-Reuter, 2010, Pollet, Glorieux et Toungouz, 2010, Boeglin et Jean, 2011, Lampron et Blaser, 2012, Reuter, 2012).

Alors, qu'est-ce que la littératie universitaire ? En quoi est-elle différente de la littératie ? Selon Delcambre et Lahanier-Reuter (2010), la littératie universitaire peut être appréhendée selon trois axes : sociologique (en ce qu'elle forme les étudiants à utiliser des formes d'écrits utiles à leur profession), cognitif (en ce qu'elle met à jour les déficits langagiers et les déficits de connaissances) et didactique (en ce qu'elle décrit les écrits pratiqués à l'université et des spécificités disciplinaires des écrits universitaires, le rapport au savoir des étudiants et les dispositifs de remédiation). Ces auteures affirment que l'objet de la littératie universitaire est le discours universitaire écrit et que cette littératie ne développe pas d'analyse multimodale de communication écrite. En effet, dans l'écriture universitaire, les discours « exigent une longue élaboration personnelle [...] et sont le résultat d'une construction individuelle, d'une confrontation à une discipline à travers des objets, des méthodologies, des corps de savoirs spécifiques » (Delcambre et Lahanier-Reuter, 2010 : 12). Reuter (2012) stipule que la lecture occupe une grande place dans la littératie universitaire, place qui ne doit pas être laissée pour compte au profit de la seule écriture. La lecture et l'écriture universitaires doivent être étudiées et les difficultés qui leur sont associées, comprises. De plus, Delcambre et Lahanier-Reuter (2010) précisent que les ancrages théoriques de la littératie universitaire sont essentiellement ceux des sciences du langage et de la didactique du français, ce qui explique la place prépondérante de 
l'écrit dans ce concept. Toutefois, la littératie universitaire se distingue de la didactique du français dans la mesure où le but de cette littératie n'est pas de développer des connaissances disciplinaires spécifiques. Ces chercheuses ajoutent que la littératie universitaire ne part pas d'un point de vue idéologique - ce qui est le cas des organismes nationaux et internationaux plaçant la littératie au cœur d'une réussite basée sur l'économie du savoir - mais qu'elle fournit des données sur les représentations des étudiants et sur l'analyse des discours universitaires, ce qui rejoint les préoccupations des chercheurs mentionnés dans le paragraphe précédent.

En somme, comme le dit Reuter (2012: 162), «le terme littéra[t]ies universitaires instaure une prise de conscience qui amène une analyse critique des fonctionnements universitaires classiques ». Nous constatons également que la littératie universitaire fait écho à la plupart des valeurs ajoutées proposées par Hébert et Lépine (2012). En effet, le concept est pluri-objectifs (valeur 1), car la littératie universitaire propose une perspective interdisciplinaire sur les objectifs et enjeux professionnels liés à l'appropriation de l'écrit. Il touche également à un ensemble d'attitudes, de connaissances, d'habiletés et de compétences liées à l'appropriation de la culture écrite (valeur 2), ici universitaire, et à développer chez l'étudiant. La littératie universitaire fait également appel à une variété de textes (valeur 3) et revêt un caractère social, dynamique et situé (valeur 4) puisque le lire-écrire à l'université représente un construit social professionnel. Elle est également synonyme de tâches scolaires réelles et authentiques (valeur 6) qui mesurent les habiletés en situations variées. La littératie universitaire représente un concept positif et un continuum (valeur 7) puisque les connaissances des étudiants en écriture universitaire évolueront tout au long de leurs études. Enfin, elle est témoin d'une visée émancipatrice (valeur 10) qui vise le développement global de l'étudiant.

\section{Questions et objectifs}

Dans ce contexte où les compétences des étudiants universitaires en littératie semblent soulever des défis importants, particulièrement pour leur maitrise des genres et des modes de discours universitaires, et où ces défis représentent aussi des enjeux certificatifs liés à la diplomation en FIE et des enjeux sociaux liés à leur future profession d'enseignant, nous en sommes venues à vouloir connaitre les mesures de remédiation mises en place dans les universités québécoises pour en comprendre l'efficacité et les limites. Nous avons donc choisi, pour cette contribution, de nous concentrer sur un cas spécifique, en poursuivant les objectifs suivants : 1) décrire des mesures d'aide en littératie universitaire en observant un cas, soit celui de l'Université du Québec en Outaouais (UQO) et de ses deux campus (Gatineau et St-Jérôme); 2) observer l'efficacité de ces mesures de remédiation en français écrit.

\section{Considérations méthodologiques}

Cette contribution est produite à la suite d'une préenquête menée dans un CAFÉ, partagé en deux campus de l'UQO. Les mesures d'aide en littératie universitaire qui sont décrites proviennent d'une collecte de données réalisées avec ce seul cas et ne sauraient faire l'objet d'une généralisation, car la situation québécoise est telle qu'il revient à chaque université de choisir d'investir, peu ou prou, dans les mesures d'aide en littératie universitaire et dans le suivi des services offerts. Ces données, qui portent sur les résultats des trois premières cohortes soumises au TECFÉE, soit celles de 2008, 2009 et 2010, constituent un échantillon de convenance fourni par l'administration de l'établissement (Département des sciences de l'éducation, UQO). Malgré 
leurs limites, elles sont utiles pour observer que des liens semblent exister entre le recours aux mesures d'aide en français écrit offert par le CAFÉ et l'augmentation du taux de réussite au TECFÉE.

\section{Les mesures d'aide observées à l'UQO}

Nous l'avons mentionné, au Québec, depuis 2008, la réussite du TECFÉE est obligatoire afin d'obtenir le brevet d'enseignant. Or, ce test présente un important taux d'échec à l'échelle nationale. L'UQO n'a pas fait exception, comme le montre le tableau suivant ${ }^{10}$ :

Tableau 1 : Première passation du TECFÉE des trois premières cohortes selon le Centre d'évaluation du rendement en français écrit ${ }^{11}$

\begin{tabular}{|c|c|}
\hline Cohortes & Taux de succès \\
\hline 2008 & $46 / 127(\mathbf{3 6} \%)$ \\
\hline 2009 & $59 / 129(\mathbf{4 6} \%)$ \\
\hline 2010 & $92 / 129(\mathbf{7 1} \%)$ \\
\hline
\end{tabular}

Les résultats de l'UQO sont représentatifs de la moyenne nationale pour ces trois premières années de passation du TECFÉE. Pour limiter les échecs et éviter d'augmenter le taux d'abandon dans leurs programmes de FIE, les universités québécoises, dont l'UQO, ont agi dans l'urgence. L'UQO a ainsi mis en place des mesures d'aide dès 2008-2009. D'ailleurs, nous posons l'hypothèse que si le taux de succès a doublé en trois ans dans cette université, c'est que des mesures d'aide ont été mises en place et qu'elles auraient une certaine efficacité.

Dans la lignée de la tradition des CAFÉ du collégial (voir section 1.2), trois formules ont été proposées aux étudiants: un cours de mise à niveau, des cours de rattrapage et des accompagnements individualisés et de groupe au CAFÉ.

- Le cours de mise à niveau est crédité et porte essentiellement sur la grammaire de la phrase. Il est assuré par un professeur ou un chargé de cours.

- Les cours de rattrapage, aussi crédités, sont plutôt orientés vers la grammaire textuelle. Ils sont assurés par un professeur ou un chargé de cours.

- L'accompagnement offert au CAFÉ n'est pas crédité et prend la forme de cinq mesures d'aide. La participation à ces mesures est volontaire et se fait en fonction des besoins des étudiants. Elles sont prises en charge par différents intervenants, selon leur nature. Ces mesures sont : 1) la consultation libre; 2) des ateliers de mise à niveau en français écrit; 3) des ateliers de préparation au TECFÉE; 4) du monitorat individuel; 5) du monitorat de groupe.

Ces cinq mesures de remédiation offertes au CAFÉ ont chacune des fonctions et des visées particulières et complémentaires.

La consultation libre est une mesure ponctuelle, utilisée de façon plutôt informelle. Les étudiants se présentent, généralement sans rendez-vous, avec une ou plusieurs questions précises ou besoins spécifiques qu'ils adressent à la professionnelle sur place (par exemple : comprendre des

${ }^{10}$ Il est impossible d'obtenir les taux de réussite des autres universités québécoises, malheureusement.

${ }^{11} \mathrm{Ce}$ centre corrige les tests de toutes les universités québécoises et fait parvenir les résultats à chaque établissement. 
erreurs identifiées dans un travail corrigé, mais incomprises par l'étudiant; connaitre des stratégies argumentatives différentes pour bonifier leurs travaux écrits; clarifier les différents usages de la virgule, etc.).

Les ateliers de mise à niveau en français écrit, offerts par la professionnelle en bloc thématique hebdomadaire de trois heures (par exemple : la ponctuation, l'accord des participes passés, les marqueurs de relation, la modalisation, etc.), adoptent la formule d'ateliers en petit groupe au cours desquels les étudiants réalisent des exercices grammaticaux structuraux tirés de divers sites Internet, de cahiers d'exercices, de matériel didactique, etc. Les étudiants choisissent d'assister $\mathrm{au}(\mathrm{x}) \operatorname{bloc}(\mathrm{s})$ thématique(s) des notions avec lesquelles ils ont davantage de difficultés. Il s'agit donc d'une mesure d'aide offerte à tous, mais dont la responsabilité du choix des thématiques à travailler revient aux étudiants.

Les ateliers de préparation au TECFÉE, offerts par la professionnelle en deux blocs thématiques (grammaire de la phrase; rédaction de compte-rendu critique), visent à former les étudiants en FIE afin qu'ils réussissent le test. Il peut autant s'agir d'étudiants qui présentent des lacunes en écriture que de scripteurs plutôt habiles qui souhaitent réviser les notions avant le test. Les principales activités réalisées sont : préparation à l'étude des notions de grammaire de la phrase (code linguistique), préparation à la rédaction (compte-rendu critique) et simulation du TECFÉE. L'objectif est d'entrainer les étudiants à réussir le test; les préoccupations ont donc des visées à court terme.

Quant au monitorat individuel, il s'agit d'une série de rencontres individuelles (une heure par semaine, pendant 10 semaines) entre un étudiant en difficulté et un moniteur (étudiant rémunéré). Pendant les rencontres, les étudiants produisent une dictée diagnostique qui cible leurs principales difficultés, travaillent sur des textes produits dans leurs cours, effectuent des exercices grammaticaux $^{12}$, etc. Le monitorat individuel permet d'accompagner les étudiants en lien avec leurs besoins spécifiques et vise à consolider quelques aspects de leur compétence scripturale.

Enfin, le monitorat de groupe est semblable aux ateliers de mise à niveau, mais les activités, animées par la professionnelle, ont lieu dans un cadre plus informel, par exemple : des séances organisées à partir des questions des étudiants, du travail fait en groupe, des dictées zéro faute pour discuter de ses (in)compréhensions, etc.

À la suite de cette recension, nous avons poursuivi nos observations afin de voir si la combinaison de ces différentes mesures permettait aux étudiants d'améliorer leur français écrit pour réussir le TECFÉE. Globalement, à partir du moment où les étudiants qui n'avaient pas réussi à la première passation du TECFÉE (voir tableau 1) recouraient à l'une des mesures de remédiation décrites ci-haut, une augmentation du taux de réussite au TECFÉE était observée :

Tableau 2 : Taux de réussite au TECFÉE après plus d'une passation selon le Centre d'évaluation du rendement en français écrit

\footnotetext{
${ }^{12}$ Tirés notamment du site Internet http://www.ccdmd.qc.ca/fr/ ou inspirés de La grammaire nouvelle (Nadeau et Fisher, 2006).
} 


\begin{tabular}{|l|l|}
\hline Cohortes & Taux de réussite $^{13}$ \\
\hline 2008 & $111 / 119(\mathbf{9 3 \%})-$ après quatre passations \\
\hline 2009 & $109 / 130(\mathbf{8 4 \%})-$ après deux passations \\
\hline 2010 & $92 / 129(\mathbf{7 1 \%})-$ après la première passation \\
\hline
\end{tabular}

La cohorte 2008

Après avoir échoué une première fois, les étudiants de la cohorte 2008 se sont principalement tournés vers deux mesures de remédiation: un cours de rattrapage crédité orienté vers la grammaire textuelle et le CAFÉ. Pour ces étudiants, qui ont vécu un taux de succès aussi faible que $36 \%$, les premières mesures de remédiation leur ont été proposées dans l'urgence avant la deuxième passation. Il faut dire qu'aucun d'entre eux n'avaient suivi de cours de mise à niveau ou de rattrapage avant la première passation et qu'une douzaine d'entre eux avaient suivi la première version des ateliers de mise à niveau offerts par le CAFÉ (quatre blocs de trois heures, une semaine avant la passation). Les deux mesures proposées, le cours de rattrapage crédité et le CAFÉ, ont été bien reçues de la part des étudiants et quelques-uns s'y sont inscrits.

En considérant les deux campus, $35 \%$ des étudiants inscrits au cours de rattrapage ont réussi la deuxième passation et $28 \%$ des étudiants qui n'étaient pas inscrits ont aussi réussi. De plus, $85 \%$ des étudiants de cette cohorte ont amélioré leur résultat à la rédaction et $70 \%$ ont amélioré leur résultat au code linguistique. Ces observations nous mènent vers deux réflexions. D'une part, il semblerait qu'un cours de rattrapage puisse être une mesure de remédiation efficace en littératie universitaire dans la mesure où la très grande majorité des étudiants ont obtenu de meilleurs résultats au test. D'autre part, il nous importe d'être prudentes dans les interprétations avancées, car ces étudiants en étaient à leur deuxième passation, de sorte qu'ils connaissaient mieux la composition du test (même si les questions n'étaient pas les mêmes) et avaient pu mieux s'y préparer, sachant à quoi s'attendre.

La cohorte 2009

Pour la cohorte de 2009, les mesures de remédiation offertes ont été bonifiées après l'expérience de la cohorte 2008. Au campus de St-Jérôme, $80 \%$ des étudiants ont suivi le cours de mise à niveau, le cours de rattrapage ou les deux et $55 \%$ ont participé aux ateliers de mise à niveau (3 heures par semaine, pendant les 10 semaines avant la passation) et au monitorat individuel au CAFÉ (1 heure par semaine pendant 10 semaines). Au campus de Gatineau, $67 \%$ des étudiants ont suivi le cours de mise à niveau, le cours de rattrapage ou les deux, et $35 \%$ ont participé aux ateliers de mise à niveau et au monitorat individuel au CAFÉ.

De ces étudiants qui ont eu recours à l'une ou l'autre des mesures de remédiation, une très grande majorité, soit $82 \%$, a réussi le test. Ces observations nous amènent à penser, d'une part, que les mesures de remédiation bonifiées (combinaison d'un cours crédité et de mesures d'aide offertes au CAFÉ, augmentation du nombre d'ateliers de mise à niveau, étalement sur 10 semaines plutôt que sur une semaine avant la passation) outillent mieux les étudiants et contribuent à

\footnotetext{
${ }^{13} \mathrm{Au}$ fil des ans, les modalités de passation du TECFÉE changent selon les exigences du Centre d'évaluation du rendement en français écrit, ce qui fut le cas en 2008 et en 2009. Pour 2010, les étudiants n'avaient fait qu'un seul test au moment de la collecte des données.
} 
l'augmentation du taux de réussite. D'autre part, il nous est impossible, compte tenu des limites de ces observations, de connaitre les mesures de remédiation les plus efficaces non seulement pour la réussite du TECFÉE, mais pour les compétences langagières des étudiants et leur maitrise de la littératie universitaire. Cependant, l'approche plus globale mise en place a peut-être permis aux étudiants de se questionner de différentes façons sur un ensemble plus vaste de connaissances et de compétences liées à la langue et à l'écriture.

La cohorte 2010

Pour la cohorte de 2010, les mesures de remédiation ont été stabilisées et uniformisées dans le temps et dans leur organisation. Ainsi, tous les étudiants ayant échoué à la suite d'une première passation ont obligatoirement dû suivre un cours de rattrapage (3h par semaine pendant 15 semaines) et une formation offerte par le CAFÉ (ateliers de mise à niveau de 3 heures par semaine pendant 15 semaine et ateliers de rédaction de comptes-rendus critiques de 3 heures par semaine pendant 5 semaines) lors du trimestre qui a précédé la nouvelle passation.

Une fois leur formation terminée, tous ces étudiants ayant suivi la formation du CAFÉ ont réussi le TECFÉE $(n=37)$, à l'exception d'un seul. Ces nouvelles observations tendent à confirmer que les mesures de remédiation offertes aux étudiants sont efficaces. Par contre, les données disponibles ne permettent pas de savoir lesquelles ont des effets plus importants. Nous posons toutefois l'hypothèse, à la suite de Lefrançois, Laurier, Lazure et Claing (2008), que l'offre combinée de différentes mesures a des effets positifs, entre autres, sur la réussite au TECFÉE.

\section{Conclusions}

Nos conclusions sont de deux ordres. D'abord, nos observations portent à croire que les mesures de remédiation, notamment lorsqu'offertes en combinaison, aident à améliorer la compétence scripturale. Nous croyons que cette combinaison s'inscrit dans une perspective de littératie universitaire, en ce sens qu'un travail intensif sur différents aspects de la langue (la connaissance des règles de grammaire) et de l'écriture (la compétence à écrire des textes) prépare mieux les étudiants en FIE à rencontrer les attentes de la société québécoise à l'égard des futurs enseignants. D'autres recherches plus poussées sur ce sujet sont nécessaires, mais nous croyons qu'elles pourraient révéler d'autres changements chez les étudiants concernés, notamment au sujet de leur rapport à l'écrit.

Ensuite, nos observations soulèvent l'importance de la littératie universitaire en tant que champ de recherche, notamment dans une perspective linguistique. Améliorer notre connaissance de ce qui constitue cette littératie, dont la connaissance des difficultés des étudiants dans leur rapport au langage en général, dans leur rapport aux genres et aux modes d'écrits universitaires et la connaissance des moyens de remédier aux difficultés de cet ordre, permettrait de mieux intervenir auprès des étudiants universitaires. Il nous apparait dès lors incontournable de produire des données de recherche pour connaitre non seulement le rapport à l'écrit d'étudiants en contexte universitaire (ce qui comprend tant leurs pratiques d'écriture que de lecture et d'oralité), mais aussi la formation qui leur est offerte en littératie universitaire. Somme toute, il ne fait nul doute qu'une meilleure connaissance des mesures de remédiation en littératie universitaire et de leurs effets permettra de proposer des dispositifs de formation adaptés aux étudiants, non seulement 
afin qu'ils réussissent une épreuve de certification nationale comme le TECFÉE, mais aussi, et surtout, afin qu'ils aient une solide maitrise des genres et des modes de discours universitaires.

\section{Bibliographie}

BARBIER, P., 2009, « Modalités et modes de communication des discours universitaires », dans J.-M. Defays, A. Englebert (dir.), Principes et typologie des discours universitaires, Paris, L'Harmattan, p. 37-46.

BEAUDET C., REY, V., 2012, «De l'écrit universitaire à l'écrit professionnel: comment favoriser le passage de l'écriture heuristique et scientifique à l'écriture professionnelle », Revue Scripta, 16-30, p. 169-193.

BEAUDRY, N., CAMERLAIN, L., BEGIN, C., 2007, « Le RUSAF s'affiche », Correspondance, 13-1. 〈http://www.ccdmd.qc.ca /correspo/Corr13-1/Rusaf.html>

BOEGLIN, M. et JEAN, A., 2011, «L'écriture en sciences de l'éducation : information, formation, transformation », Les cahiers du CERFEE, 30. <http://www.lirdef.univmontp2.fr/cerfee/spip.php?article473>

CARTIER, S., LANGEVIN, L., 2001, «Tendances et évaluations des dispositifs de soutien aux étudiants du postsecondaire dans le Québec francophone », Revue des sciences de l'éducation, XXVII-2, p. 353-381.

CLAING, R., 2007, «Le Centre d'aide en français du collège Ahuntsic. Les moments clés de son histoire », Correspondance, 12-4. 〈http://correspo.ccdmd.qc.ca/Corr12-4/Ahuntsic.html>

COGHLAN, V., THERIAULT, J.-Y., 2002, L'apprentissage du français en milieu minoritaire, une revue documentaire, Ottawa, Centre interdisciplinaire de recherche sur la citoyenneté et les minorités et Fédération canadienne des enseignantes et des enseignants.

CONSEIL CANADIEN SUR L'APPRENTISSAGE, 2011, Quel est le futur de l'apprentissage au Canada? Toronto : Conseil canadien de l'apprentissage.

DELCAMBRE, I., LAHANIER-REUTER, D., 2010, Les littéracies universitaires: Influence des disciplines et du niveau d'étude dans les pratiques de l'écrit < www.formulecture.ch>

DORE, T., DEZUTTER, O., 2006, «Le rapport à l'écriture d'étudiantes et d'étudiants en formation initiale à l'enseignement : terrain propice à un nouveau dispositif écrit de formation? », dans R. Bélisle, S. Boudon (dir.), Pratiques et apprentissage de l'écrit dans les sociétés éducatives, Québec, Les Presses de l’Université Laval, p. 223-246.

FEDERATION DES CEGEPS. 1999, La réussite et la diplomation au collégial: des chiffres et des engagements, Montréal, Fédération des cégeps.

FEDERATION DES SYNDICATS DE L'ENSEIGNEMENT, 2011, Les droits, pouvoirs et responsabilités des enseignantes et des enseignants, Québec, FSE.

GRENIER, S., JONES, S., STRUCKER, J., MURRAY, T.S, GERVAIS, G., BRINK, S., 2008, International Adult Literacy Survey. Learning Literacy in Canada: Evidence from the International Survey of Reading Skills, Ottawa (Canada), Statistique Canada et Ministère de l'Industrie.

HARPER, J., STEIN DZALDOV, B., 2012, Literacy Smarts: Simple Classroom Strategies for Using Interactive Whiteboards to Engage Students, Ottawa, Pembroke Publishing.

HART-DAVIDSON, W., GRABILL, J. T., 2011, «Understanding and Supporting Knowledge work in Schools, Workplaces, and Public Life », dans D. Starke-Meyerring, A. Paré, N. Artemeva, M. Horne, L. Yousoubova (dir.), Writing in Knowledge Societies, Fort Collins, Colorado, The WAC Clearinghouse and Parlor Press, p. 161-176. 
HEBERT, M., LEPINE, M., 2012, « Analyse et synthèse des principales définitions de la notion de littératie en francophonie ", Lettrure, 2 <http://www.ablf.be/lettrure/lettrure-2/analyse-etsynthese-des-principales-definitions-de-la-notion-de-litteratie-en-francophonie>

LABASSE, B., 2006, La communication écrite: une matière en quête de substance, Lyon, Éditions Colbert \& CECP Éditions.

LAMPRON, R., BLASER, C., 2012, "La maîtrise des compétences langagières dans la formation : regard sur les pratiques étatsuniennes », Correspondance, 18-1, p. 7-11.

LEFRANÇOIS, P., LAURIER, M.D., LAZURE, R., CLAING, R., 2008, Évaluation de l'efficacité des mesures visant l'amélioration du français écrit du primaire à l'université, Rapport de recherche. Montréal, Université de Montréal.

MARTINEAU, S., PRESSEAU, A. 2003, «Le sentiment d'incompétence pédagogique des enseignants en début de carrière et le soutien à l'insertion professionnelle », Brock Education Journal, 12-2, p. 54-67.

MOFFET, J.-D., DELMASY, A. 1994, Les compétences et la maitrise du français au collégial. Rapport de recherche PAREA. Rimouski, Cégep de Rimouski.

MOREAU, A.C., HEBERT, M., LEPINE, M., RUEL, J. 2013, «Le concept de littératie en francophonie: que disent les définitions? », Revue Consortium national de recherche sur l'intégration sociale, 4-2, p. 14-18.

NADEAU, M., FISHER, C., 2006, La grammaire nouvelle, la comprendre et l'enseigner, Montréal, Chenelière.

NADEAU, M. 2010, «Réflexion sur les exigences du TECFÉE et les besoins langagiers des futurs enseignants », Communication présentée au Colloque du Réseau universitaire des services d'aide en français (RUSAF), Montréal, juin.

ORGANISATION DE COOPERATION ET DE DEVELOPPEMENT ECONOMIQUES [OCDE], 2004, Apprendre aujourd'hui, réussir demain. Premiers résultats de PISA 2003, Paris, OCDE.

ORGANISATION DE COOPERATION ET DE DEVELOPPEMENT ECONOMIQUES [OCDE], 2011, Enseignement supérieur et apprentissage des adultes. Regards sur l'éducation 2011 : Les indicateurs de l'OCDE, Paris, OCDE.

POLlET, M.-C., GLORIEUX, C., TOUNGOUZ, K., 2010, «Pour un continuum dans l'appropriation d'une littéracie universitaire », Diptyque, 18, p. 61-92.

RÉSEAU CANADIEN DE RECHERCHE SUR LE LANGAGE ET L'ALPHABÉTISATION, 2009, Stratégie nationale d'alphabétisation précoce. London (Canada), <http://docs.cllrnet.ca/NSEL/finalReportFR.pdf>

REUTER, Y. 2012, «Les didactiques et la question des littéracies universitaires », Pratiques, 153-154, p. 161-176. 